\title{
Effects of modified LDL and HDL on retinal pigment epithelial cells: a role in diabetic retinopathy?
}

\author{
M. Du • M. Wu • D. Fu • S. Yang • J. Chen • K. Wilson • T. J. Lyons
}

Received: 19 February 2013 / Accepted: 17 June 2013 / Published online: 11 July 2013

(C) Springer-Verlag Berlin Heidelberg 2013

\begin{abstract}
Aims/hypothesis Blood-retina barrier leakage in diabetes results in extravasation of plasma lipoproteins. Intra-retinal modified LDLs have been implicated in diabetic retinopathy (DR), but their effects on retinal pigment epithelial (RPE) cells and the added effects of extravasated modified HDLs are unknown.

Methods In human retinas from individuals with and without diabetes and DR, immunohistochemistry was used to detect ApoB, ApoA1 and endoplasmic reticulum (ER) stress markers. In cell culture, human RPE cells were treated with native LDL (N-LDL) or heavily-oxidised glycated LDL (HOG-LDL) with or without pretreatment with native HDL (N-HDL) or heavilyoxidised glycated HDL (HOG-HDL). Cell viability, oxidative stress, ER stress, apoptosis and autophagy were assessed by Cell Counting Kit-8 assay, dichlorofluorescein assay, western blotting, immunofluorescence and TUNEL assay. In separate experiments, RPE cells were treated with lipid oxidation products, 7-ketocholesterol $(7-\mathrm{KC}, 5-40 \mu \mathrm{mol} / \mathrm{l})$ or 4 hydroxynonenal (4-HNE, 5-80 $\mu \mathrm{mol} / \mathrm{l})$, with or without pretreatment with N-HDL or HOG-HDL.
\end{abstract}

Electronic supplementary material The online version of this article (doi:10.1007/s00125-013-2986-x) contains peer-reviewed but unedited supplementary material, which is available to authorised users.

M. Du $\cdot \mathrm{M} . \mathrm{Wu} \cdot \mathrm{D} . \mathrm{Fu} \cdot \mathrm{S}$. Yang $\cdot \mathrm{J}$. Chen $\cdot \mathrm{K}$. Wilson

T. J. Lyons $(\bowtie)$

Harold Hamm Diabetes Center and Section of Endocrinology

and Diabetes, University of Oklahoma Health Sciences Center,

HHDC Suite 2900, Oklahoma City, OK 73104, USA

e-mail: timothy-lyons@ouhsc.edu

M. Du

Department of Physiology, University of Oklahoma Health

Sciences Center, Oklahoma City, OK, USA

D. Fu

Department of Immunology, Harbin Medical University,

Harbin, People's Republic of China
Results ApoB, ApoA1 staining and RPE ER stress were increased in the presence of DR. HOG-LDL but not N-LDL significantly decreased RPE cell viability and increased reactive oxygen species generation, ER stress, apoptosis and autophagy. Similarly, 4-HNE and 7-KC decreased viability and induced ER stress. Pretreatment with N-HDL mitigated these effects, whereas HOG-HDL was less effective by most, but not all, measures.

Conclusions/interpretation In DR, extravascular modified LDL may promote RPE injury through oxidative stress, ER stress, autophagy and apoptosis. N-HDL has protective effects, but HOG-HDL is less effective. Extravasation and modification of HDL may modulate the injurious effects of extravasated modified LDL on the retinal pigment epithelium.

Keywords Apoptosis - Autophagy - Diabetic retinopathy · ER stress $\cdot$ HDL $\cdot$ LDL

$\begin{array}{ll}\text { Abbreviations } & \\ \text { ATF6 } & \text { Activating transcription factor } 6 \\ \text { BRB } & \text { Blood-retina barrier } \\ \text { CCK-8 } & \text { Cell Counting Kit-8 } \\ \text { CHOP } & \text { C/EBP-homologous protein } \\ \text { Cyt-C } & \text { Cytochrome } c \\ \text { DCF } & \text { Dichlorofluorescein } \\ \text { DR } & \text { Diabetic retinopathy } \\ \text { ER } & \text { Endoplasmic reticulum } \\ \text { GPX-1 } & \text { Glutathione peroxidase 1 } \\ \text { GRP78 } & \text { 78 kDa glucose-regulated protein } \\ \text { 4-HNE } & \text { 4-Hydroxynonenal } \\ \text { HOG-HDL } & \text { Highly oxidised glycated HDL } \\ \text { HOG-LDL } & \text { Highly oxidised glycated LDL } \\ \text { hTERT-RPE } & \text { Telomerase-immortalised human RPE } \\ \text { 7-KC } & \text { 7-Ketocholesterol } \\ \text { LC3 } & \text { Microtubule-associated protein } \\ & \text { 1 light chain 3 } \\ \text { NDRI } & \text { National Disease Research Interchange }\end{array}$




$\begin{array}{ll}\text { N-HDL } & \text { Native HDL } \\ \text { N-LDL } & \text { Native LDL } \\ \text { Ox-LDL } & \text { Oxidised LDL } \\ \text { PARP } & \text { Poly ADP ribose polymerase } \\ \text { ROS } & \text { Reactive oxygen species } \\ \text { RPE } & \text { Retinal pigment epithelial } \\ \text { RPE65 } & \text { Retinal pigment epithelium-specific } \\ & \text { 65 kDa protein } \\ \text { SFM } & \text { Serum-free medium } \\ \text { SOD-2 } & \text { Superoxide dismutase 2 }\end{array}$

\section{Introduction}

Diabetic retinopathy (DR) is the leading cause of acquired blindness among working-age adults [1]. The main risk factors include duration of diabetes, long-term severity of hyperglycaemia and hypertension [2], but other factors, including dyslipoproteinaemia [3, 4], are also implicated.

Dyslipoproteinaemia is an established risk for atherogenesis in people with and without diabetes. The term extends beyond quantitative measures and includes 'post-synthetic' modifications of plasma lipoproteins (e.g. by glycation and oxidation). Such modifications may occur in plasma but predominantly occur in tissues following lipoprotein extravasation and sequestration (e.g. in the arterial intima) $[5,6]$. In diabetes, modification is enhanced by elevated glucose, increased oxidative stress and prolongation of exposure resulting from enhanced crosslinking to matrix proteins [7, 8]. Modified lipoproteins are considered central to the pathogenesis of atherosclerosis, wherein their harmful effects occur predominantly at the site of disease (i.e. in tissue, not in plasma).

The retina is a highly specialised tissue and extravasation of plasma constituents, including lipoproteins, is normally prevented by the inner and outer blood-retina barriers (BRBs) [9]. In DR, BRB leakage is an early feature. We hypothesised that plasma lipoproteins play a 'hidden' but important role in DR, critically dependent upon the presence and extent of BRB leakage and thus not primarily determined by, or related to, dyslipoproteinaemia. In support, we demonstrated immunostaining of ApoB100 (the principal apolipoprotein of LDL) and oxidised LDL (ox-LDL) in retinas of people with diabetes, even in the absence of clinical DR, and among those with DR to a greater extent and proportional to disease severity [9]. In early DR, immunostaining for ox-LDL was initially localised near the inner BRB (i.e. in the ganglion cell layer) while in more advanced DR it permeated all layers of the retina [9]. No immunostaining for $\mathrm{ApoB}$ or ox-LDL was found in retinas from people without diabetes.

Epidemiological data are consistent with an indirect link between dyslipoproteinaemia and DR: there are highly significant, yet relatively weak, associations between plasma lipoprotein levels and DR severity $[3,10,11]$. The weakness of these associations has, we contend, led to a lack of appreciation of the important role lipoproteins play in propagating DR, one that is only operative after BRB leakage, but significant regardless of plasma lipoprotein concentrations.

Further support for our hypothesis comes from previous cell culture work documenting the effects of modified LDL on retinal capillary endothelial cells [12] and pericytes [9, 13-16]. We showed that the mechanisms of LDL-mediated pericyte injury include oxidative and endoplasmic reticulum (ER) stresses, apoptosis and autophagy [16]. We have also demonstrated deleterious effects of modified LDL on retinal Muller cells [17], suggesting a retinal insult that extends beyond vascular cells.

Accumulating evidence suggests that early in DR, barrier leakage affects not only the inner but also the outer BRB [18]. The outer BRB is comprised of tight junctions between retinal pigment epithelial (RPE) cells. The retinal pigment epithelium is a multi-functional cell monolayer and is critically important to retinal health. Exposure of the retinal pigment epithelium to modified LDL could therefore mediate significant retinal injury, including leakage of the outer BRB.

The present study employs human tissues for immunochemistry and cell culture studies on the effects of modified LDL on human RPE cells in culture and, since extravasation of LDL implies extravasation of HDL and also since extravasated HDL is subject to the same modifying stresses as LDL, we also studied the effects of normal and modified HDL. HDL has particular relevance to the retinal pigment epithelium. There is extensive cholesterol transport across the RPE monolayer and intra-retinal 'HDL-like particles' mediate export of cholesterol from retinal rods and cones to the retinal pigment epithelium [19]. Two important products of lipid peroxidation, 7-ketocholesterol (7-KC) and 4-hydroxynonenal (4-HNE), were also employed to confirm findings and to define the utility of these compounds as surrogates for modified LDL in future studies.

\section{Methods}

Ethics The study was approved by the Institutional Review Board at the University of Oklahoma Health Sciences Center.

Human retinal tissues Normal human eyes and eyes from individuals with diabetes, fixed in $10 \%$ neutral buffered formalin within $12 \mathrm{~h}$ of death, were obtained from the $\mathrm{Na}$ tional Diseases Research Interchange (NDRI; Philadelphia, PA, USA). Three groups (eyes from three individuals per group) were included in the study: without diabetes, with 
type 2 diabetes but without retinopathy and type 2 diabetes with retinopathy. Ophthalmological records provided DR status and the absence of other retinal diseases, including macular oedema. Eyes from individuals with diabetic neuropathy or nephropathy were excluded. Paraffin-embedded retinal sections $(5 \mu \mathrm{m})$ were prepared and used for immunohistochemistry studies.

Human lipoproteins: preparation, modification and characterisation of LDL and HDL Native LDL (N-LDL) and modified LDL were prepared as previously described [9, 20], and native HDL (N-HDL) and modified HDL were prepared using similar protocols. Briefly, human LDL and HDL were isolated from pooled plasma obtained from male and female healthy volunteers ( $n=4$ for each preparation), aged $20-40$ years, who were not taking antioxidant vitamins or any prescribed medications. N-LDL and N-HDL were isolated by sequential ultracentrifugation $(350,000 \mathrm{~g}$, N-LDL density [d] $=1.019$ 1.063 , N-HDL density [d] $=1.063-1.21)$ of pooled plasma. 'Highly oxidised glycated' (HOG-) LDL and HOG-HDL were prepared by first glycating N-LDL or N-HDL in the presence of freshly prepared $50 \mathrm{mmol} / 1 \mathrm{D}$-glucose for $72 \mathrm{~h}$ at $37^{\circ} \mathrm{C}$ under antioxidant conditions $(1 \mathrm{mmol} / 1$ diethylenetriaminepentaacetic acid (DTPA), $270 \mu \mathrm{mol} / 1$ EDTA, under nitrogen). The glycated lipoproteins were oxidised by incubation in $10 \mu \mathrm{mol} / 1 \mathrm{CuCl}_{2}\left(24 \mathrm{~h}, 37^{\circ} \mathrm{C}\right)$, followed by extensive dialyses to remove copper ions and glucose [20]. Protein content was determined (BCA protein assay; Pierce, Rockford, IL, USA). Lipoproteins were characterised by gel electrophoresis (Paragon Lipo Gel; Beckman, Fullerton, CA, USA), by fluorescence (360 nm excitation $/ 430 \mathrm{~nm}$ emission; Gilford Fluorometer IV; Oberlin, $\mathrm{OH}, \mathrm{USA}$ ) and by absorbance at $234 \mathrm{~nm}$ (DU650 spectrophotometer; Beckman). The protocols aimed to simulate the initial in vivo glycation of lipoproteins in plasma and their subsequent oxidation after extravasation and sequestration in tissues. Lipoprotein preparations were stored in the dark under nitrogen at $4^{\circ} \mathrm{C}$, and were used within 1 month of preparation. Experiments were repeated using different LDL preparations.

Human RPE cell cultures Telomerase-immortalised human RPE (hTERT-RPE) cells (ATCC, Manassas, VA, USA) were cultured in DMEM containing $4.5 \mathrm{~g} / 1$ glucose, $10 \% \mathrm{FCS}$, $100 \mathrm{U} / \mathrm{ml}$ penicillin and $100 \mu \mathrm{g} / \mathrm{ml}$ streptomycin. Cells were grown to $80-90 \%$ confluence and were made quiescent by overnight exposure to serum-free medium (SFM) before the addition of lipoproteins.

Immunohistochemistry in human diabetic retinas Immunostaining of ApoB, ApoA1, ER stress marker $78 \mathrm{kDa}$ glucoseregulated protein (GRP78; Lys-Asp-Glu-Leu) and retinal pigment epithelium-specific $65 \mathrm{kDa}$ protein (RPE65) in human retinas was performed as described [9]. Briefly, retinal sections were incubated with primary anti-ApoB (1:100), anti-ApoA1 (1:100) and anti-GRP78 (1:50) (Abcam, Cambridge, MA, USA) or anti-RPE65 (1:100; Millipore, Billerica, MA, USA) $\left(4^{\circ} \mathrm{C}\right.$, overnight $)$, then incubated with secondary antibodies $\left(37^{\circ} \mathrm{C}, 2 \mathrm{~h}\right)$. Immunostaining for ox-LDL and 4-HNE employed primary antibodies from Abcam. Fluorescence signals were visualised under a fluorescence microscope (Nikon E800 Epifluorescence Microscope, Tokyo, Japan).

Cell viability assay hTERT-RPE cells were cultured in 96-well plates $(15,000$ cells/well). Cells were exposed to $\mathrm{N}$ - or HOG-LDL $(200 \mu \mathrm{g}$ protein/ml), 7-KC $(5-40 \mu \mathrm{mol} / \mathrm{l})$ or 4-HNE $(5-80 \mu \mathrm{mol} / \mathrm{l})$, with or without pretreatment with $\mathrm{N}$ - or HOG-HDL $(500 \mu \mathrm{g} / \mathrm{ml})$. Cell viability was measured by Cell Counting Kit- 8 assay (CCK-8; Dojindo Molecular Technologies, Rockville, MD, USA) according to the manufacturer's instructions. Briefly, treated cells were washed with SFM, incubated with CCK-8 solution $\left(2 \mathrm{~h}, 37^{\circ} \mathrm{C}\right)$ and absorbance was measured at $450 \mathrm{~nm}$.

Immunocytochemistry: activating transcription factor 6 translocation and TUNEL assay RPE cells were cultured to $80 \%$ confluence on glass coverslips, then treated with $\mathrm{N}$ or HOG-LDL $(200 \mu \mathrm{g} / \mathrm{ml})$ for $12 \mathrm{~h}$, with or without pretreatment with N- or HOG-HDL $(500 \mu \mathrm{g} / \mathrm{ml})$. After fixation (4\% paraformaldehyde fixation buffer, $15 \mathrm{~min}$ ) and permeabilisation (0.5\% Triton X-100 in PBS, 15 min), cells were incubated with anti-ATF6 (Abcam) (overnight, $4^{\circ} \mathrm{C}$ ), then with Alexa Fluor 594-conjugated anti-rabbit $\operatorname{IgG}(2 \mathrm{~h}$, room temperature). Fluorescence was visualised using a Nikon E800 Epifluorescence Microscope.

Cell death was detected by TUNEL assay (In Situ Cell Death Detection Kit; Roche Diagnostics, Indianapolis, IN, USA). Briefly, RPE cells grown on glass coverslips were incubated with TUNEL reaction mixture, counterstained with DAPI and positive nuclei were identified (bright green signal).

Dichlorofluorescein assay Intracellular reactive oxygen species (ROS) were measured using a dichlorofluorescein (DCF) kit (Invitrogen, Carlsbad, CA, USA). RPE cells were studied following exposure to N- or HOG-LDL $(200 \mu \mathrm{g} / \mathrm{ml})$ for up to $1 \mathrm{~h}$, with or without pretreatment with $\mathrm{N}$ - or HOG-HDL $(500 \mu \mathrm{g} / \mathrm{ml}, 1 \mathrm{~h})$. Briefly, cells were incubated with $10 \mu \mathrm{mol} / 12^{\prime}, 7^{\prime}$-dichlorodihydrofluorescein diacetate solution $\left(37^{\circ} \mathrm{C}, 30 \mathrm{~min}\right)$ before N-LDL treatment. DCF fluorescence was detected by a microplate reader (excitation: $485 \mathrm{~nm}$; emission: $538 \mathrm{~nm}$ ) (VICTOR3 V Multilabel Counter; PerkinElmer Life and Analytical Sciences, Waltham, MA, USA) and expressed as a ratio of baseline levels.

Western blotting RPE cells were treated with N- or HOG-LDL (200 $\mu \mathrm{g} / \mathrm{ml}), 7-\mathrm{KC}(10 \mu \mathrm{mol} / \mathrm{l})$ or 4 -HNE $(20 \mu \mathrm{mol} / \mathrm{l})$ for 
$12 \mathrm{~h}$, with or without pretreatment with N- or HOG-HDL $(500 \mu \mathrm{g} / \mathrm{ml}, 1 \mathrm{~h})$. Cells were washed with PBS, lysed (RIPA lysis buffer; Santa Cruz Biotechnology, Santa Cruz, CA, USA) and protein extracts $(30 \mu \mathrm{g})$ were run on $12 \%$ SDS-PAGE gels, then transferred onto nitrocellulose filter membranes (Pall Life Sciences, Port Washington, NY, USA). Membranes were incubated with specific primary antibodies and detected with horseradish peroxidase-conjugated secondary antibodies. Probed proteins were visualised (Super Signal ELISA Femto Maximum Sensitivity Substrate; Thermo Scientific, Rockford, IL, USA) and detected (BioSpectrum Imaging System; UVP, Upland, CA, USA). Band intensities were normalised to $\beta$-actin and quantified using UVP analysing software (VisionWorksLS Image Acquisition and Analysis Software, UVP).

Data analysis Data are presented as mean $\pm \mathrm{SE}$ of at least three independent experiments. Differences between groups were examined using one-way or two-way ANOVA followed by Dunnett's test. A $p$ value of $<0.05$ was considered statistically significant.

\section{Results}

Detection of ApoB and ApoA1 in retinas from humans with and without diabetes and $D R$ To determine whether extravasated LDL is present in the vicinity of the retinal pigment endothelium, we performed double-staining for ApoB and RPE65 in retinal sections from individuals with type 2 diabetes with and without DR, and from non-diabetic individuals (Fig. 1a). To detect HDL, we performed immunostaining for ApoA1, the principal apolipoprotein of HDL (Fig. 1b). Consistent with our previous reports [9], ApoB staining was negligible in non-diabetic retinas but was present in diabetic retinas, especially in the presence of DR. Furthermore, in retinas from individuals with $\mathrm{DR}$, increased $\mathrm{ApoB}$ staining was observed immediately adjacent to the retinal pigment epithelium most clearly on its apical aspect, but also on the basolateral side (Fig. 1a). The staining of ApoA1 (Fig. 1b) was almost entirely in the vicinity of the retinal pigment epithelium in non-diabetic retinas, but in diabetic eyes, it was observed in the inner retina and in the presence of DR, there was widespread immunostaining throughout the retina.

$H O G$-LDL decreases RPE cell viability: protection by $N-H D L$ but not HOG-HDL Exposure of RPE cells to HOG-LDL (50, $100,200,300 \mu \mathrm{g} / \mathrm{ml}$ ) reduced cell viability compared with SFM or N-LDL (200 $\mu \mathrm{g} / \mathrm{ml})$ (Fig. 2a). In time course studies, HOG-LDL $(200 \mu \mathrm{g} / \mathrm{ml})$ reduced RPE cell viability by $12 \mathrm{~h}$ (Fig. 2b). Pretreatment of cells with N-HDL (Fig. 2c) but not HOG-HDL (Fig. 2d) for $1 \mathrm{~h}$ prevented this effect. The



Fig. 1 Presence of ApoB and ApoA1 in the human retina. Representative retinal images for (a) ApoB (green) and RPE65 (red) and (b) ApoA1 staining (red) from five non-diabetic individuals, three individuals with type 2 diabetes with no DR and three individuals with type 2 diabetes with non-proliferative DR (NPDR). ApoA1 was detected in $\mathrm{RPE}$ layers in all three groups. It was also present in the inner retina of diabetic individuals and was widely distributed in those with NPDR. Magnification: (a) $\times 40$, (b) $\times 20$; scale bar, $100 \mu \mathrm{m}$. INL, inner nuclear layer; GCL, ganglion cell layer; RPE, retinal pigment epithelium; ONL, outer nuclear layer

protective effect of N-HDL was dose-dependent, whereas pretreatment with HOG-HDL amplified the toxic effect of HOG-LDL. HOG-HDL alone did not decrease viability. The effects of LDL and HDL on viability were visualised by microscopy (Fig. 2e-h).

HOG-LDL induces RPE cell apoptosis: inhibition by N-HDL but not HOG-HDL Cells were exposed to N-LDL or HOG-LDL $(200 \mu \mathrm{g} / \mathrm{ml}, 12 \mathrm{~h})$, with or without pretreatment with N-HDL or HOG-HDL $(500 \mu \mathrm{g} / \mathrm{ml}, 1 \mathrm{~h})$. Apoptosis, detected by TUNEL assay, was markedly increased by HOG-LDL vs $\mathrm{N}-\mathrm{LDL}$, and was mitigated by pretreatment with N-HDL but not HOG-HDL (Fig. 3a). Expression of cytochrome c (Cyt-C) was markedly increased by HOG- LDL vs N-LDL, and this increase was ameliorated by N-HDL pretreatment, but not by HOG-HDL (Fig. 3b). In contrast, cleaved poly-(ADP ribose)-polymerase (PARP) was only slightly affected by the lipoproteins (Fig. 3c). 



Fig. 2 HOG-LDL reduced RPE cell viability: protection by N-HDL but not HOG-HDL. RPE cells were cultured in 96-well plates to $80 \%$ confluence then made quiescent by exposure to SFM overnight. (a) Dose-dependent changes of viability in RPE cells treated with HOG-LDL for $24 \mathrm{~h}$. (b) Time course of $200 \mu \mathrm{g} / \mathrm{ml} \mathrm{HOG-LDL-induced} \mathrm{RPE} \mathrm{cell}$ viability loss. RPE cells were pretreated with (c) N-HDL or (d) HOG-HDL (both $500 \mu \mathrm{g} / \mathrm{ml}$ ) for $1 \mathrm{~h}$, then treated with $200 \mu \mathrm{g} / \mathrm{ml} \mathrm{HOG-LDL}$ for $24 \mathrm{~h}$. Cell viability was determined by CCK-8 assay. Data are presented as mean $\pm \mathrm{SE}\left({ }^{*} p<0.05\right.$ vs SFM; ${ }^{\dagger} p<0.05$ vs HOG-LDL). Cell density changes of RPE cells treated with (e) N-LDL or (f) HOG-LDL (both $200 \mu \mathrm{g} / \mathrm{ml}$ ) for $24 \mathrm{~h}$, with and without pretreatment with (g) N-HDL or (h) HOG-HDL (both $500 \mu \mathrm{g} / \mathrm{ml}$ ) for $1 \mathrm{~h}$. Images were obtained using phasecontrast microscope. Data are representative of three independent experiments. Magnification: $\times 10$; scale bar, $60 \mu \mathrm{m}$

HOG-LDL increases intracellular ROS and decreases glutathione peroxidase 1 expression in RPE cells: inhibition by $N$-HDL but not HOG-HDL Treatment of RPE cells for up to 60 min with HOG-LDL vs N-LDL significantly increased the production of ROS (Fig. 4a, b) and decreased the expression of glutathione peroxidase 1 (GPX-1) (Fig. 4c, d). Pretreatment with N-HDL dramatically reduced the ROS ratio in HOG-LDL-treated cells, and HOG-HDL was less effective (Fig. 4b). In the presence of HOG-LDL, N-HDL significantly increased GPX-1 expression whereas HOG-HDL had no effect (Fig. 4d). Superoxide dismutase 2 (SOD-2) expression (western blots) was unaffected by the lipoproteins (data not shown).

HOG-LDL induces ER stress: inhibition by N-HDL but not $H O G-H D L$ ER stress was determined by expression of GRP78, CCAAT/enhancer-binding protein homologous protein (CHOP), phosphorylation of eIF2 $\alpha$ and nuclear translocation of activating transcription factor 6 (ATF6). Representative western blots are shown in Fig. 5a, b and data from triplicate experiments in Fig. 5c-h. HOG-LDL vs N-LDL $(200 \mu \mathrm{g} / \mathrm{ml})$ increased GRP78 and CHOP expression and eIF2 $\alpha$ phosphorylation (Fig. 5a, c-e). Pretreatment with N-HDL blocked HOG-LDL-induced ER stress, but HOG-HDL only partially inhibited CHOP expression and eIF $2 \alpha$ phosphorylation (Fig. $5 b, \mathrm{f}-\mathrm{h}$ ). Translocation of ATF6 (cytoplasm to nucleus) was induced by HOG-LDL but not $\mathrm{N}-\mathrm{LDL}$ at $12 \mathrm{~h}$, and was prevented by pretreatment with N-HDL but not HOG-HDL (Fig. 5i).

HOG-LDL induces autophagy: inhibition by both N-HDL and HOG-HDL During autophagosome formation, conversion of unconjugated soluble microtubule-associated protein 1 light chain 3 (LC3)-I to its conjugated form, LC3-II, is a marker of autophagy. HOG-LDL vs N-LDL $(200 \mu \mathrm{g} / \mathrm{ml}, 12$ $24 \mathrm{~h}$ ) increased LC3-II expression (Fig. 6a). Pretreatment with either N-HDL or HOG-HDL $(500 \mu \mathrm{g} / \mathrm{ml}, 1 \mathrm{~h})$ inhibited HOG-LDL-induced LC3-II expression (Fig. 6b). Expression of another autophagy-related protein, Beclin-1, was not altered by HOG-LDL or N-LDL, or by pretreatment with N-HDL or HOG-HDL (western blots in Fig. 6a, b).

GRP78 expression is increased in retinal pigment epithelium of retinas from individuals with $D R$ vs non-DR diabetic or non-diabetic individuals To define the clinical relevance of our in vitro findings, we performed double-staining for GRP78 and RPE65 in retinas from diabetic individuals with and without DR and from non-diabetic individuals (Fig. 7). GRP78 staining was very faint and homogenous in retinas from non-diabetic individuals and from diabetic individuals without DR, but was increased in diabetic retinas in the presence of DR (Fig. 7). Merged images (yellow) revealed partial co-localisation of GRP78 in the retinal pigment epithelium layer, suggesting a role for ER stress in RPE cells in the pathogenesis of DR.

7-KC and 4-HNE decreased RPE cell viability and induced ER stress: protection by $N-H D L$ and partial protection by $H O G-H D L$ LDL is a large particle and during oxidation numerous products are generated, including $7-\mathrm{KC}$ and 4-HNE. We treated RPE cells with these specific lipid oxidation products and the results are shown in electronic supplementary materials (ESM) Figs 1, 2. Both 7-KC and 4-HNE decreased RPE cell viability in a dose-dependent manner (ESM Fig. 1) and increased levels of the ER stress markers p-eIF $2 \alpha$, X-box binding protein-1 and CHOP (ESM Fig. 2). The effects of 7-KC and 4-HNE were effectively mitigated by pretreatment with N-HDL but less so by HOG-HDL.

Detection of ox-LDL and 4-HNE in human retinas We observed increased staining for ox-LDL and 4-HNE in diabetic 
Fig. 3 HOG-LDL induced apoptosis in RPE cells: protection by N-HDL but not HOG-HDL. Confluent RPE cells were exposed to N-LDL or HOG-LDL (both $200 \mu \mathrm{g} / \mathrm{ml}$ ) for $12 \mathrm{~h}$, with and without pre-incubation with N-HDL or HOG-HDL (both $500 \mu \mathrm{g} / \mathrm{ml}$ ) for $1 \mathrm{~h}$. (a) Representative images of TUNEL staining. Apoptotic RPE cells were revealed by TUNEL-positive labelling (green) and nuclei were counterstained with DAPI (magnification: $\times 40$; scale bar, $20 \mu \mathrm{m})$. (b, c) Cell lysates were assessed for apoptotic markers Cyt-C and cleaved-PARP by western blot analysis; $\beta$-actin was loaded as protein control. Data are presented as mean $\pm \mathrm{SE}$ $\left({ }^{*} p<0.05\right.$ vs SFM; ${ }^{\dagger} p<0.05$ vs HOG-LDL)
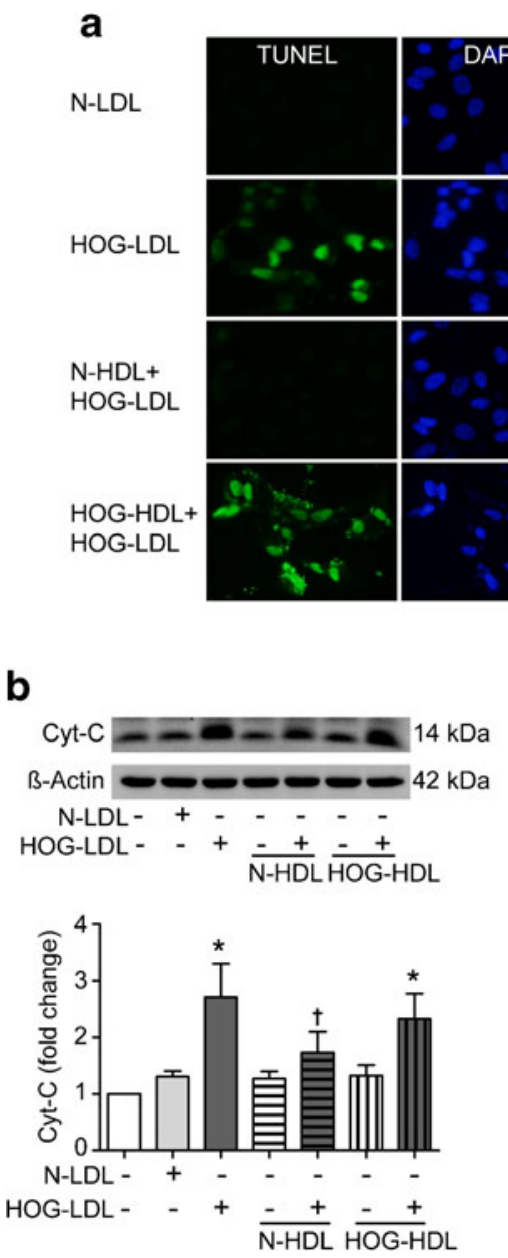

C
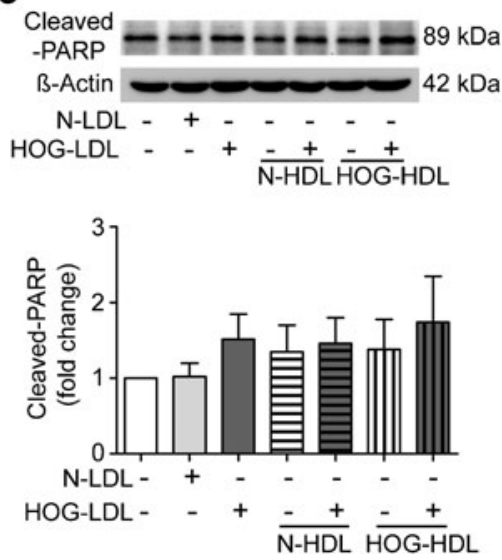

retinas in the vicinity of the retinal pigment epithelium, more marked in the presence of clinical DR (ESM Fig. 3).

\section{Discussion}

The present study provides further support for our hypothesis that plasma lipoproteins play a central role in the propagation of DR, but one that is mainly operative after BRB leakage has led to lipoprotein extravasation and modification. We contend that modified lipoproteins then mediate toxicity towards many different retinal cell types, both vascular and non-vascular. In support of this, we previously identified the presence of extensive amounts of modified LDL in the diabetic retina and we defined its toxicity towards retinal capillary pericytes [9, 13-15], endothelial cells [12] and Muller cells [17].

This study is the first to address the effects of modified LDL on the retinal pigment epithelium, and to investigate the role of extravasated modified HDL. In human diabetic retinas, we demonstrate increased presence of ApoB adjacent to the retinal pigment epithelium on both sides, augmenting previous findings [9]. Supporting an involvement of HDL, we demonstrate increased ApoA1 staining in diabetic retinas, consistent with HDL extravasation. Such staining in diabetic retina was previously demonstrated by Sima et al [21] and was accompanied by increased expression of mRNA. This was attributed to increased production by the retinal pigment epithelium and we contend that HDL leakage is also likely to contribute. Furthermore, we demonstrate the presence of ox-LDL and 4-HNE in the vicinity of the retinal pigment epithelium in DR, using immunohistochemistry (ESM Fig. 3). We also demonstrate RPE ER stress in the presence of DR, consistent with effects of modified LDL in our cell culture studies.

In cell culture, we show that modified LDL is toxic towards RPE cells, consistent with mediation of outer BRB injury and with recent evidence that not only the inner but also the outer BRB is compromised in DR [18]. We define mechanisms for the effects of modified LDL on the retinal pigment epithelium, and show that native HDL is protective, whereas modified HDL is, in general, not protective.

Our overall hypothesis draws analogies with the role of ox-LDL in atherosclerosis, but with important differences. 

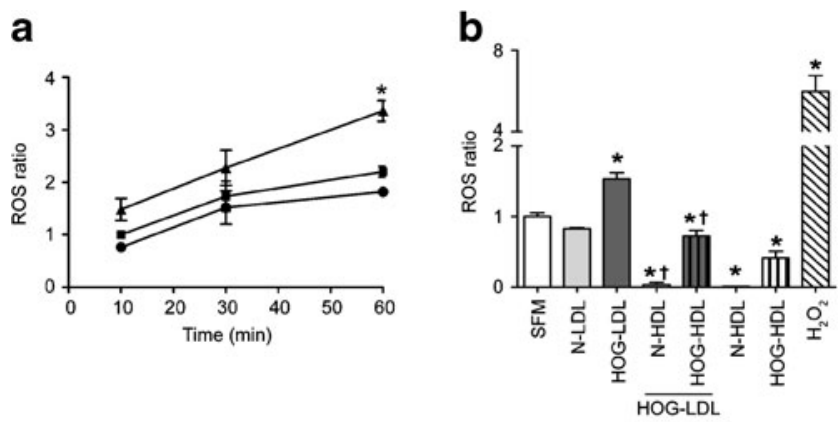

C
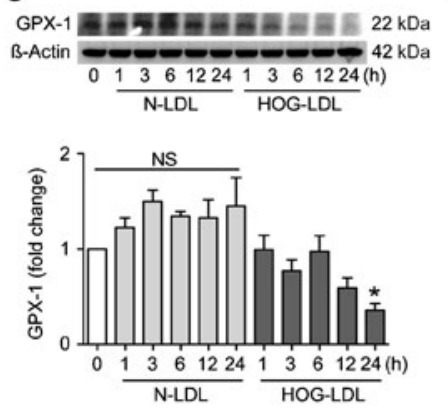

d


Fig. 4 HOG-LDL induced ROS production and reduced antioxidant enzyme GPX-1 expression in RPE cells: prevention by N-HDL but not HOG-HDL. ROS were measured by DCF fluorescence. Results were expressed as ratio of baseline SFM condition. (a) Confluent RPE cells were exposed to N-LDL or HOG-LDL (both $200 \mu \mathrm{g} / \mathrm{ml}$ ) for 10, 30 and $60 \min \left({ }^{*} p<0.05\right.$ vs SFM). Triangle, HOG-LDL; square, SFM; circle, N-LDL. (b) RPE cells were pre-incubated with N-HDL or HOG-HDL (both $500 \mu \mathrm{g} / \mathrm{ml}$ ) for $1 \mathrm{~h}$, followed by co-incubation with HOG-LDL $(200 \mu \mathrm{g} / \mathrm{ml})$ for $1 \mathrm{~h}\left({ }^{*} p<0.05 \mathrm{vs} \mathrm{SFM} ;{ }^{\dagger} p<0.05\right.$ vs HOG-LDL $) . \mathrm{H}_{2} \mathrm{O}_{2}$ $(1 \mathrm{mmol} / \mathrm{l})$ was used as positive control. (c) HOG-LDL $(200 \mu \mathrm{g} / \mathrm{ml})$ reduced GPX-1 expression in RPE over $24 \mathrm{~h}$. (d) Using concentrations of LDL and HDL as in (b), the HOG-LDL-induced reduction of GPX-1 expression was mitigated by $\mathrm{N}$ - but not by HOG-HDL. Data are presented as mean $\pm \mathrm{SE}\left({ }^{*} p<0.05\right.$ vs $\mathrm{SFM} ;{ }^{\dagger} p<0.05$ vs HOG-LDL)

First, in arteries, LDL extravasation begins in childhood [22] whereas in healthy retina, lipoprotein extravasation is rigorously prevented throughout life. Thus, with both BRBs intact, dyslipoproteinaemia may be largely irrelevant to retinal function, but once BRB breakdown occurs, as in diabetes, the 'fold increase' in extravasated lipoproteins between diabetic vs healthy retina may greatly exceed that in arterial intima. Second, the retina differs markedly in structure from the artery. The retinal 'neuropile' is embryologically part of the brain [23] and although only approximately $0.5 \mathrm{~mm}$ thick, comprises a complex assembly of specialised cell types with negligible interstitial space: both BRBs are immediately adjacent to neurons and neural support cells. The retina consumes more oxygen per gram [24] and has a higher blood flow $[25,26]$ than any other tissue; it also contains a high concentration of unsaturated, oxidisable lipids. In DR, the protection normally provided by the intact BRB is compromised, exposing many different retinal cell types to modified, cytotoxic plasma lipoproteins.
Leakage of the inner BRB is well-recognised in DR and recent work by ourselves [9] and others [18] suggests that the outer BRB is also compromised. The outer BRB comprises tight junctions between cells of the RPE monolayer, and separates the choroidal circulation from the neural retina. The retinal pigment epithelium performs numerous other functions critical to intra-retinal homeostasis [27, 28], including control of cholesterol flux [29,30]. We now show that HOG-LDL but not N-LDL causes apoptosis of RPE cells, and that this effect is mediated by oxidative stress, ER stress and autophagy. The injurious effects of HOG-LDL were ameliorated by pretreatment with native HDL; pretreatment with HOG-HDL was less effective and sometimes enhanced toxicity (e.g. decreased cell viability compared with HOG-LDL alone). As in previous work with retinal capillary pericytes and Muller cells [9,13,15-17], we endeavoured to ensure that cell culture conditions (LDL concentration, extent of modification) simulated stresses present in vivo in human DR.

We demonstrate that in RPE cells, HOG-LDL induces ROS production and decreases levels of GPX-1. Increased oxidative stress may damage susceptible lipids, proteins and other biomolecules and is a recognised mediator of atherosclerosis [31, 32], diabetes [33] and (as found in our own recent work) pericyte injury in DR $[16,17]$. GPX-1 is the most important cytosolic and mitochondrial antioxidant enzyme in humans. It detoxifies hydrogen peroxide and lipid peroxides and its decreased activity is associated with increased risk for cardiovascular disease and diabetes [34, 35]. HDL has known antioxidant effects and in the present study of RPE cells, N-HDL completely suppressed the HOG-LDL-induced increase in ROS, but HOG-HDL was much less effective.

The ER serves vital functions, including lipid and protein synthesis, maturation and transportation, and 'ER stress' is linked to major diseases including cancer, neurodegenerative diseases and diabetes [36]. ER stress, mediated by excessive fatty acid flux, may induce protein misfolding and trigger the 'unfolded protein response' [37]. In the diabetic eye, ER stress has been implicated in the death of retinal neurons and vascular cells [38]. The ER is sensitive to oxidative stress induced by exposure to ox-LDL, as shown in our studies of retinal pericytes $[16,17]$. In the present study, ER stress was induced by HOG-LDL in human RPE cells, and this effect was inhibited by pretreatment with N-HDL but only partially by HOG-HDL. Prolonged ER stress ultimately results in cell apoptosis via activation of the pro-apoptotic transcription factor CHOP and in our study HOG-LDL dramatically induced CHOP expression. Both $\mathrm{N}$ - and HOG-HDL inhibited the increase in CHOP expression.

Autophagy plays a key role in maintaining the intracellular environment, removing damaged organelles, cell membranes and proteins, and preserving nutrients for energy [39]. It may be induced by ER stress, which in turn it 
Fig. 5 ER stress was involved in HOG-LDL-induced RPE cell injury: protection by N-HDL and partial protection by HOG-HDL. (a, b) Representative western blots performed on total protein extracts are shown. (a) Time course of ER stress protein expression in RPE cells treated with N-LDL or HOG-LDL (200 $\mu \mathrm{g} / \mathrm{ml})$. (b) RPE cells were pretreated with $\mathrm{N}-\mathrm{HDL}$ or HOG-HDL $(500 \mu \mathrm{g} / \mathrm{ml})$ for $1 \mathrm{~h}$, followed by co-incubation with HOG-LDL $(200 \mu \mathrm{g} / \mathrm{ml})$ for $12 \mathrm{~h}$. (c-e) Quantification of blots from triplicate experiments as in (a). (f-h) Quantification of blots from triplicate experiments as in (b). (i) Representative images of cytoplasmic staining and nuclear translocation of ATF6 in RPE cells treated with N-LDL or HOG-LDL $(200 \mu \mathrm{g} / \mathrm{ml})$ for $12 \mathrm{~h}$, with and without pre-incubation with N-HDL or HOG-HDL $(500 \mu \mathrm{g} / \mathrm{ml})$ for $1 \mathrm{~h}$ (magnification: $\times 40$; scale bar, $20 \mu \mathrm{m}$ )

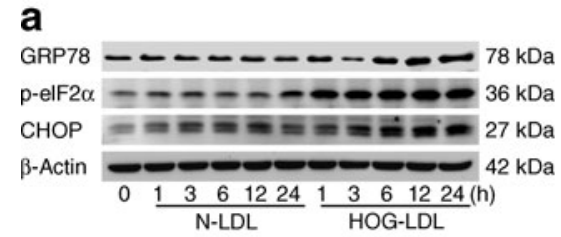

b

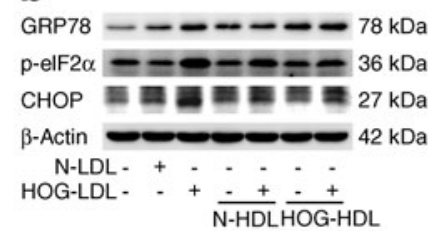

C

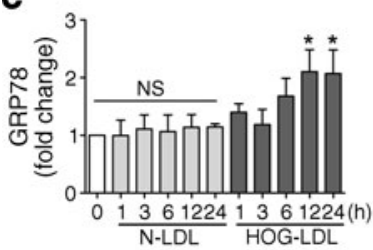

d


f
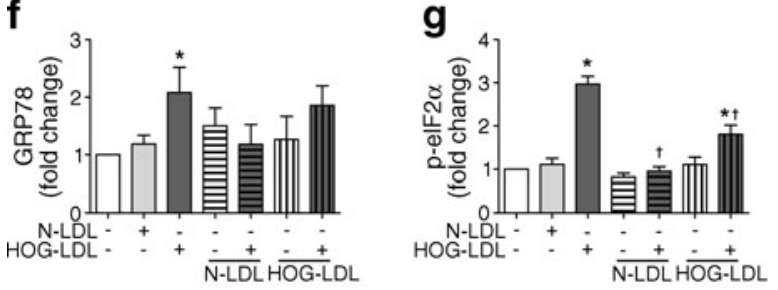

h
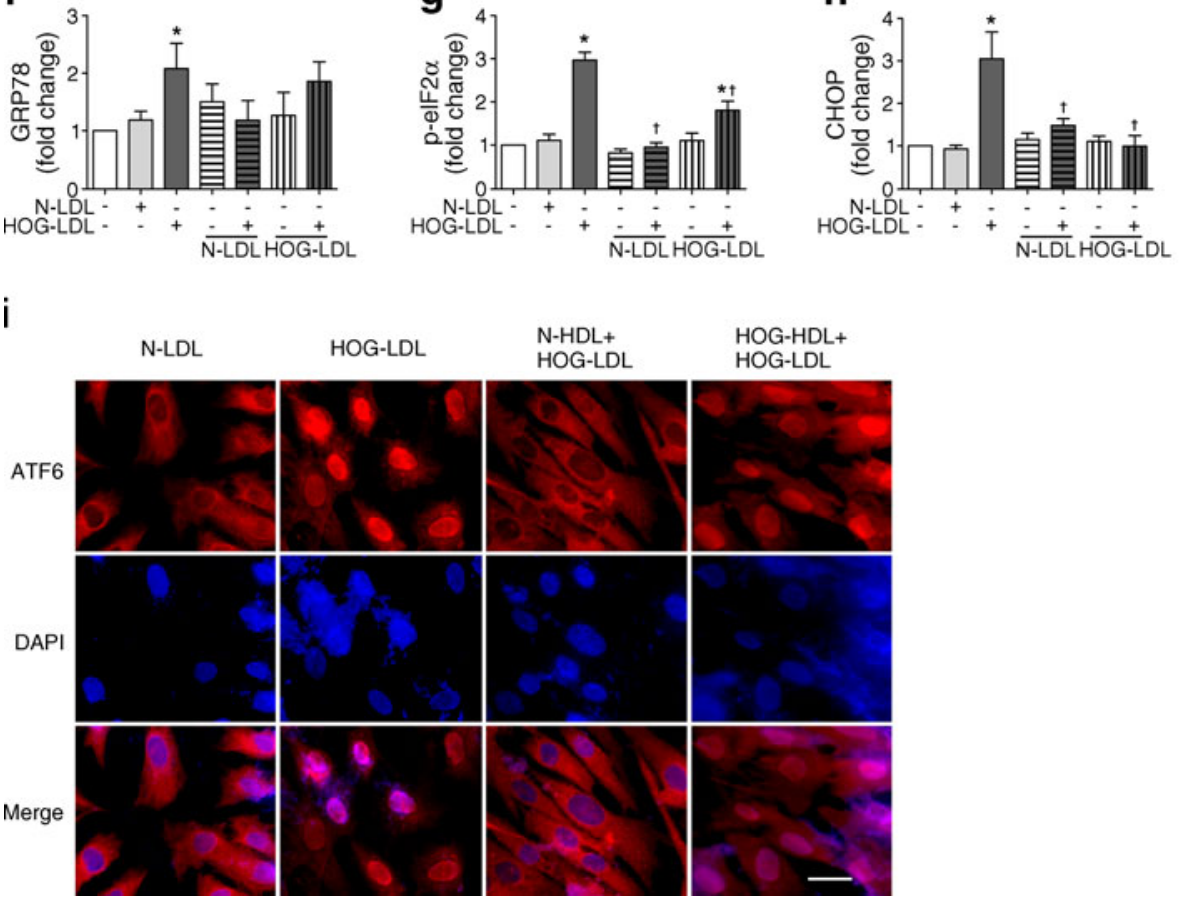

may alleviate by promoting degradation of damaged proteins [40]. Prolonged ER stress may switch autophagy from a protective role to one promoting cell death. Recent evidence implicates autophagy in the intracellular degradation of oxLDL in human vascular endothelial cells [41]. We have shown that autophagy mediates the effects of HOG-LDL on retinal pericytes and Muller cells [16, 17]. In the present work, we show that treatment of RPE cells with HOG-LDL promotes conversion of LC3 from its cytosolic (LC3-I) to its membranebound form (LC3-II), an essential step in autophagosome formation. Not only N-HDL but also HOG-HDL prevents this effect. The mechanisms whereby both N-HDL and HOG-HDL can block CHOP activation and prevent the activation of autophagy need further study, and this emphasises the great complexity and multiple functions of HDL [42].
It is reported that in atherosclerosis ox-LDL triggers Cyt-C release, leading to caspase activation and vascular cell apoptosis, thereby promoting plaque formation [43]. We have documented HOG-LDL-induced apoptosis in retinal pericytes and Muller cells $[9,16,17]$, and our present results with RPE cells are analogous: HOG-LDL induces apoptosis through upregulation of pro-apoptotic Cyt-C and PARP. N-HDL protects RPE cells from HOG-LDL-induced apoptosis by blocking expression of these proteins, but HOG-HDL is ineffective.

HDL is generally considered to be atheroprotective by removing excess cholesterol from cells, inhibiting lipid oxidation and inhibiting inflammatory responses [44-46]. Qualitative changes in HDL occur in diabetes, including enhanced glycation of ApoA1 molecules [47], and these may impair HDL function. For example, the capacity of HDL for reverse cholesterol transport is reduced by glycation [48] or oxidation 
a
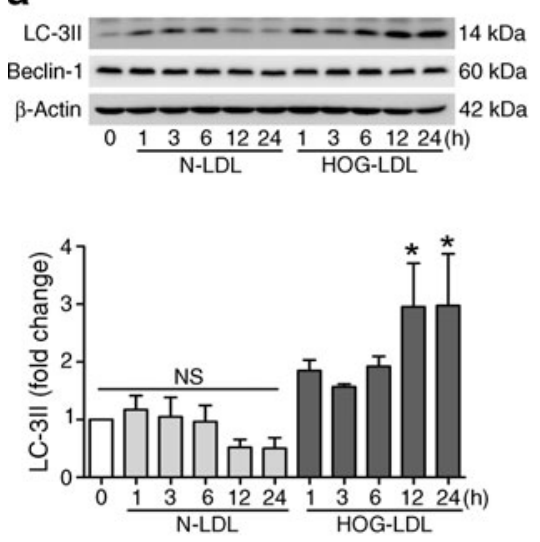

Fig. 6 HOG-LDL increased autophagy in RPE cells: mitigation by Nand HOG-HDL. (a) Time course of LC3-II expression in RPE cells treated with N-LDL or HOG-LDL $(200 \mu \mathrm{g} / \mathrm{ml})$. (b) RPE cells were pretreated with N-HDL or HOG-HDL $(500 \mu \mathrm{g} / \mathrm{ml})$ for $1 \mathrm{~h}$, followed by

[49]. Consistent with this, the anti-atherogenic properties of HDL isolated from the plasma of individuals with diabetes are impaired compared with HDL isolated from non-diabetic individuals [50]. This implies that once BRB leakage occurs, the



Fig. 7 Detection of GRP78 in retinal sections from non-diabetic human subjects, type 2 diabetes with and without diabetic retinopathy. Human retinal sections were stained with antibody against GRP78 (green) and RPE65 (red), and the nuclei were counterstained with DAPI (blue). Images were representative of three independent experiments. Yellow in merged images represents overlapping of green and red signals, indicating increased expression of GRP78 in human RPE layer. Magnification: $\times 60$; scale bar, $20 \mu \mathrm{m}$. INL, inner nuclear layer; ONL, outer nuclear layer; RPE, retinal pigment epithelium b
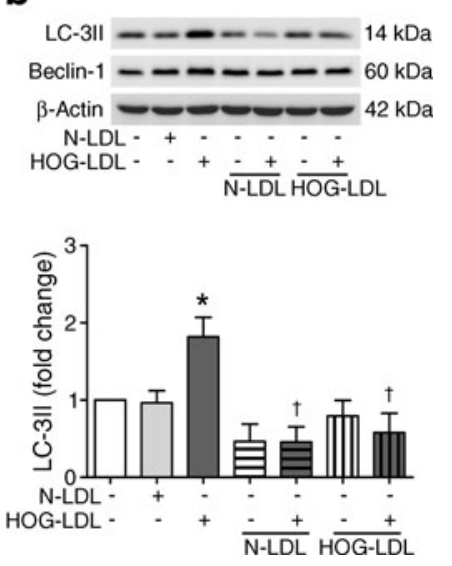

HOG-LDL $(200 \mu \mathrm{g} / \mathrm{ml})$ treatment for $12 \mathrm{~h}$. Western analysis were performed on total protein extracts and blots were quantified relative to control $\left({ }^{*} p<0.05\right.$ vs SFM; ${ }^{\dagger} p<0.05$ vs HOG-LDL)

diabetic retina suffers a double insult from both LDL and HDL: not only do the lipoproteins gain access to a space from which they are normally excluded but also the quality of those lipoproteins is already compromised by glycation in plasma, and is further compromised by subsequent modification.

Finally, we tested the effects of two model compounds of lipoprotein oxidation, 7-KC and 4-HNE. These are much more readily studied than modified LDL and had effects on RPE cells that were generally similar to those of HOG-LDL. They may serve as useful surrogates for HOG-LDL, at least for exploratory experiments.

In conclusion, we demonstrate that HOG-LDL may cause oxidative stress, ER stress and autophagy in RPE cells, thus mediating RPE cell apoptosis, and potentially contributing to compromise of the outer BRB. In DR, immunostaining for ApoB shows its presence in the vicinity of the retinal pigment epithelium, and for ApoA1 suggests extravasation of HDL. N-HDL completely blocked the oxidative stress, ER stress, autophagy and apoptosis induced by HOG-LDL. Modification of HDL, first by glycation then by oxidation, abolished some but not all its protective properties, and by one measure rendered it actively toxic, emphasising the structural and metabolic complexity of this particle. Overall, the findings suggest an important new role for extravasated and modified plasma lipoproteins in promoting DR: they may compromise the many important functions of the retinal pigment epithelium, including the integrity of the outer BRB. Future studies should define the differential effects of modified LDL on the apical and baso-lateral aspects of the retinal pigment epithelium. Improved understanding of these disease mechanisms may lead to new strategies to treat and prevent the development and progression of DR.

Acknowledgements The NDRI provided valued assistance in obtaining human retinal tissues. 
Funding This work was supported by the COBRE Program of the National Center for Research Resources (P20 RR 024215), the American Diabetes Association (07-12-CT-46), the LINJO fund and by the Oklahoma Center for the Advancement of Science and Technology (HR08-067).

Duality of interest The authors declare that there is no duality of interest associated with this manuscript.

Contribution statement MD contributed to the conception and design of the study and to acquisition of data and analysis, interpretation of data and the drafting of the paper. MW and JC contributed to the conception and design of the study and to revision of the paper. DF, SY and $\mathrm{KW}$ contributed to the acquisition of data and revision of the paper. TJL contributed to the conception and design of the study and to writing and revision of the paper. All authors gave approval of the final version to be published.

\section{References}

1. Fong DS, Aiello LP, Ferris FL 3rd, Klein R (2004) Diabetic retinopathy. Diabetes Care 27:2540-2553

2. West KM, Erdreich LJ, Stober JA (1980) A detailed study of risk factors for retinopathy and nephropathy in diabetes. Diabetes 29:501-508

3. Lyons TJ, Jenkins AJ, Zheng D et al (2004) Diabetic retinopathy and serum lipoprotein subclasses in the DCCT/EDIC cohort. Investig Ophthalmol Vis Sci 45:910-918

4. Klein R, Sharrett AR, Klein BE et al (2002) The association of atherosclerosis, vascular risk factors, and retinopathy in adults with diabetes : the atherosclerosis risk in communities study. Ophthalmology 109:1225-1234

5. Matsuura E, Kobayashi K, Tabuchi M, Lopez LR (2006) Oxidative modification of low-density lipoprotein and immune regulation of atherosclerosis. Prog Lipid Res 45:466-486

6. Steinberg D, Parthasarathy S, Carew TE, Khoo JC, Witztum JL (1989) Beyond cholesterol. Modifications of low-density lipoprotein that increase its atherogenicity. N Engl J Med 320:915-924

7. Lyons TJ, Baynes JW, Patrick JS, Colwell JA, Lopes-Virella MF (1986) Glycosylation of low density lipoprotein in patients with type 1 (insulin-dependent) diabetes: correlations with other parameters of glycaemic control. Diabetologia 29:685-689

8. Ravandi A, Kuksis A, Shaikh NA (2000) Glucosylated glycerophosphoethanolamines are the major LDL glycation products and increase LDL susceptibility to oxidation: evidence of their presence in atherosclerotic lesions. Arterioscler Thromb Vasc Biol 20:467-477

9. Wu M, Chen Y, Wilson K et al (2008) Intraretinal leakage and oxidation of LDL in diabetic retinopathy. Investig Ophthalmol Vis Sci 49:2679-2685

10. van Leiden HA, Dekker JM, Moll AC et al (2002) Blood pressure, lipids, and obesity are associated with retinopathy: the hoorn study. Diabetes Care 25:1320-1325

11. Lloyd CE, Klein R, Maser RE, Kuller LH, Becker DJ, Orchard TJ (1995) The progression of retinopathy over 2 years: the Pittsburgh Epidemiology of Diabetes Complications (EDC) Study. J Diabetes Complications 9:140-148

12. Lyons TJ, Li W, Wells-Knecht MC, Jokl R (1994) Toxicity of mildly modified low-density lipoproteins to cultured retinal capillary endothelial cells and pericytes. Diabetes 43:1090-1095

13. Song W, Barth JL, Lu K et al (2005) Effects of modified low-density lipoproteins on human retinal pericyte survival. Ann N Y Acad Sci 1043:390-395

14. Song W, Barth JL, Yu Y et al (2005) Effects of oxidized and glycated LDL on gene expression in human retinal capillary pericytes. Investig Ophthalmol Vis Sci 46:2974-2982
15. Diffley JM, Wu M, Sohn M, Song W, Hammad SM, Lyons TJ (2009) Apoptosis induction by oxidized glycated LDL in human retinal capillary pericytes is independent of activation of MAPK signaling pathways. Mol Vis 15:135-145

16. Fu D, Wu M, Zhang J et al (2012) Mechanisms of modified LDL-induced pericyte loss and retinal injury in diabetic retinopathy. Diabetologia 55:3128-3140

17. Wu M, Yang S, Elliott MH et al (2012) Oxidative and endoplasmic reticulum stresses mediate apoptosis induced by modified LDL in human retinal Muller cells. Investig Ophthalmol Vis Sci 53:4595-4604

18. Xu HZ, Le YZ (2011) Significance of outer blood-retina barrier breakdown in diabetes and ischemia. Investig Ophthalmol Vis Sci 52:2160-2164

19. Tserentsoodol N, Gordiyenko NV, Pascual I, Lee JW, Fliesler SJ, Rodriguez IR (2006) Intraretinal lipid transport is dependent on high density lipoprotein-like particles and class B scavenger receptors. Mol Vis 12:1319-1333

20. Jenkins AJ, Velarde V, Klein RL et al (2000) Native and modified LDL activate extracellular signal-regulated kinases in mesangial cells. Diabetes 49:2160-2169

21. Simo R, Garcia-Ramirez M, Higuera M, Hernandez C (2009) Apolipoprotein A1 is overexpressed in the retina of diabetic patients. Am J Ophthalmol 147(319-325):e311

22. Holman RL, Mc GH Jr, Strong JP, Geer JC (1958) The natural history of atherosclerosis: the early aortic lesions as seen in New Orleans in the middle of the of the 20th century. Am J Pathol 34:209-235

23. Curcio CA, Johnson M, Huang JD, Rudolf M (2010) Apolipoprotein B-containing lipoproteins in retinal aging and age-related macular degeneration. J Lipid Res 51:451-467

24. Yu DY, Cringle SJ (2001) Oxygen distribution and consumption within the retina in vascularised and avascular retinas and in animal models of retinal disease. Prog Retin Eye Res 20:175-208

25. Friedman E, Kopald HH, Smith TR (1964) Retinal and choroidal blood flow determined with krypton- 85 anesthetized animals. Investig Ophthalmol 3:539-547

26. Feke GT, Tagawa H, Deupree DM, Goger DG, Sebag J, Weiter JJ (1989) Blood flow in the normal human retina. Investig Ophthalmol Vis Sci 30:58-65

27. Steinberg RH (1985) Interactions between the retinal pigment epithelium and the neural retina. Doc Ophthalmol 60:327-346

28. Strauss $O(2005)$ The retinal pigment epithelium in visual function. Physiol Rev 85:845-881

29. Gordiyenko N, Campos M, Lee JW, Fariss RN, Sztein J, Rodriguez IR (2004) RPE cells internalize low-density lipoprotein (LDL) and oxidized LDL (oxLDL) in large quantities in vitro and in vivo. Investig Ophthalmol Vis Sci 45:2822-2829

30. Zheng W, Reem RE, Omarova S et al (2012) Spatial distribution of the pathways of cholesterol homeostasis in human retina. PLoS One 7:e37926

31. White CR, Brock TA, Chang LY et al (1994) Superoxide and peroxynitrite in atherosclerosis. Proc Natl Acad Sci U S A 91:1044-1048

32. Miller FJ Jr, Gutterman DD, Rios CD, Heistad DD, Davidson BL (1998) Superoxide production in vascular smooth muscle contributes to oxidative stress and impaired relaxation in atherosclerosis. Circ Res 82:1298-1305

33. Johansen JS, Harris AK, Rychly DJ, Ergul A (2005) Oxidative stress and the use of antioxidants in diabetes: linking basic science to clinical practice. Cardiovasc Diabetol 4:5

34. Lewis P, Stefanovic N, Pete J et al (2007) Lack of the antioxidant enzyme glutathione peroxidase- 1 accelerates atherosclerosis in diabetic apolipoprotein E-deficient mice. Circulation 115:2178-2187

35. Maritim AC, Sanders RA, Watkins JB 3rd (2003) Diabetes, oxidative stress, and antioxidants: a review. J Biochem Mol Toxicol 17:24-38 
36. Lin JH, Walter P, Yen TS (2008) Endoplasmic reticulum stress in disease pathogenesis. Annu Rev Pathol 3:399-425

37. Salminen A, Kauppinen A, Hyttinen JM, Toropainen E, Kaarniranta K (2010) Endoplasmic reticulum stress in age-related macular degeneration: trigger for neovascularization. Mol Med 16:535-542

38. Oshitari T, Hata N, Yamamoto S (2008) Endoplasmic reticulum stress and diabetic retinopathy. Vasc Health Risk Manag 4:115-122

39. Rabinowitz JD, White E (2010) Autophagy and metabolism. Science 330:1344-1348

40. Ullman E, Fan Y, Stawowczyk M, Chen HM, Yue Z, Zong WX (2008) Autophagy promotes necrosis in apoptosis-deficient cells in response to ER stress. Cell Death Differ 15:422-425

41. Zhang YL, Cao YJ, Zhang X et al (2010) The autophagy-lysosome pathway: a novel mechanism involved in the processing of oxidized LDL in human vascular endothelial cells. Biochem Biophys Res Commun 394:377-382

42. Heinecke JW (2012) The not-so-simple HDL story: a new era for quantifying HDL and cardiovascular risk? Nat Med 18:1346-1347

43. Li Y, Higashi $\mathrm{Y}$, Itabe $\mathrm{H}$, Song $\mathrm{YH}$, Du J, Delafontaine $\mathrm{P}$ (2003) Insulin-like growth factor-1 receptor activation inhibits oxidized LDL-induced cytochrome $\mathrm{C}$ release and apoptosis via the phosphatidylinositol 3 kinase/Akt signaling pathway. Arterioscler Thromb Vasc Biol 23:2178-2184
44. Assmann G, Nofer JR (2003) Atheroprotective effects of highdensity lipoproteins. Annu Rev Med 54:321-341

45. Negre-Salvayre A, Dousset N, Ferretti G, Bacchetti T, Curatola G, Salvayre R (2006) Antioxidant and cytoprotective properties of high-density lipoproteins in vascular cells. Free Radic Biol Med 41:1031-1040

46. Parthasarathy S, Barnett J, Fong LG (1990) High-density lipoprotein inhibits the oxidative modification of low-density lipoprotein. Biochim Biophys Acta 1044:275-283

47. Berthezene F (1996) Non-insulin dependent diabetes and reverse cholesterol transport. Atherosclerosis 124(Suppl):S39S42

48. Nobecourt E, Davies MJ, Brown BE et al (2007) The impact of glycation on apolipoprotein A-I structure and its ability to activate lecithin:cholesterol acyltransferase. Diabetologia 50:643653

49. Sharma N, Desigan B, Ghosh S, Sanyal SN, Ganguly NK, Majumdar S (1999) The role of oxidized HDL in monocyte/macrophage functions in the pathogenesis of atherosclerosis in rhesus monkeys. Scand J Clin Lab Invest 59:215-225

50. Kalogerakis G, Baker AM, Christov S et al (2005) Oxidative stress and high-density lipoprotein function in type I diabetes and endstage renal disease. Clin Sci (Lond) 108:497-506 